\title{
Moderate-to-severe ovarian hyperstimulation syndrome: A retrospective multivariate logistic regression analysis in Chinese patients
}

\author{
Tianzhong Ma ${ }^{A, D, E}$, Yanru Niu ${ }^{C}$, Bing Wei, ${ }^{A, F}$, Lihua $X^{B}{ }^{B}$, Lin Zou ${ }^{B, D}$, Xiaoqun Che ${ }^{B}$,

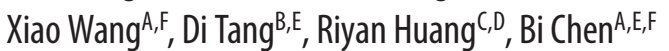 \\ Reproductive Medicine Center, Affiliated Hospital of Guangdong Medical University, Zhanjiang, China \\ A - research concept and design; $B$ - collection and/or assembly of data; $C$ - data analysis and interpretation; \\ $\mathrm{D}$ - writing the article; $\mathrm{E}$ - critical revision of the article; $\mathrm{F}$ - final approval of the article
}

Address for correspondence

Bi Chen

E-mail: chenbi2016614@sina.com

\section{Funding sources}

This study was supported by the National Nature Science Foundation of China (grant No. 81300484): Special Competitive Allocation Project of Science and Technology Special Financial Funding of Zhanjiang (grant No. 2013A1006); and the PhD Start-up Fund of Guangdong Medical College (grant No. B2012027).

Conflict of interest

None declared

Received on September 22, 2017

Reviewed on 0ctober 25, 2017

Accepted on July 4, 2018

Published online on January 28, 2020

Cite as

Ma T, Niu Y, Wei B, et al. Moderate-to-severe ovarian hyperstimulation syndrome: A retrospective multivariate logistic regression analysis in Chinese patients. Adv Clin Exp Med. 2020:29(1):85-90. doi:10.17219/acem/92916

DOI

10.17219/acem/92916

\section{Copyright}

Copyright by Author(s)

This is an article distributed under the terms of the

Creative Commons Attribution Non-Commercial License

(http://creativecommons.org/licenses/by-nc-nd/4.0/)

\section{Abstract}

Background. Ovarian hyperstimulation syndrome (OHSS), a life-threatening complication occurring in stimulated ovarian cycles, arises from treatment with gonadotropin for inducing follicular maturation.

Objectives. The aim of this study was to compare the risk factors between patients with severe OHSS and those without OHSS after in vitro fertilization by intracytoplasmatic sperm injection/embryo transfer (IVFICSI/ET). Identifying the associated risk factors may provide guidance for clinicians on how to prevent OHSS.

Material and methods. The retrospective study involved patients who had completed IVF-ICSI/ET cycles. The difference in markers for predicting the occurrence of OHSS between groups was compared. The potential protective and risk factors, as well as the predictive markers, were identified.

Results. Patients with OHSS were younger $(p=0.015)$, had higher basal antral follicle counts (AFC) $(p<0.001)$ and lower total dosages of gonadotropin $(G n)(p=0.011)$. On the day of human chorionic gonadotropin ( $(\mathrm{CG}$ ) administration, significantly higher total numbers of follicles $(p<0.001)$, serum estradiol $(E 2)(p<0.001)$ and progestrone $(P g)(p=0.001)$ levels, numbers of oocytes $(p<0.001)$ and metaphase II (MII) 0ocytes ( $p<0.001$ ) were also observed in the OHSS group when compared to the non-OHSS group. A univariate regression analysis revealed that age $(O \mathrm{R}=0.898,95 \% \mathrm{Cl}=0.822-0.981)$ and total dosage of $\mathrm{Gn}$ $(O R=0.999,95 \% \mathrm{Cl}=0.999-1.000)$ were protective factors, whereas AFC $(0 \mathrm{R}=1.090,95 \% \mathrm{Cl}=1.051-$ 1.131) and, on the day of $\mathrm{hCG}$ injection, the number of follicles $(O \mathrm{R}=1.185,95 \% \mathrm{Cl}=1.027-1.230)$, serum E2 $(O R=1.000,95 \% \mathrm{Cl}=1.000-1.000)$ and $\mathrm{Pg}(\mathrm{OR}=2.773,95 \% \mathrm{Cl}=0.510-3.370)$ levels, the number of oocytes ( $O R=1.254,95 \% \mathrm{Cl}=0.894-1.472)$ and $\mathrm{Mll}$ oocytes $(\mathrm{OR}=1.238,95 \% \mathrm{Cl}=0.747-1.217)$ were risk factors for OHSS. However, a multivariate regression analysis showed that the total number of follicles $(O R=1.124,95 \% \mathrm{Cl}=1.027-1.230)$ was the only predictive factor for the occurrence of OHSS.

Conclusions. The study demonstrated that the follicle count measured on the day of hCG administration was the only predictive factor for the occurrence of OHSS. This provides basic guidance to clinicians on the prevention of the complication when using assisted reproductive technologies (ART).

Key words: in vitro fertilization, ovarian hyperstimulation syndrome, human chorionic gonadotropin 
Ovarian hyperstimulation syndrome (OHSS), a lifethreatening complication occurring in stimulated ovarian cycles, arises from treatment with gonadotropin for inducing follicular maturation. The syndrome is commonly observed in women undergoing treatment with controlled ovarian hyperstimulation $(\mathrm{COH})$ employed during assisted reproductive technology (ART). It is characterized by a significant increase in vascular permeability and ovarian enlargement, ${ }^{1,2}$ as well as hemoconcentration with increased blood viscosity and leakage of fluid into the extravascular space. Several possible mediators, such as vascular endothelial growth factor (VEGF), reninangiotensin system and other cytokines, may be involved the development of this complication. ${ }^{3}$ Patients with severe OHSS may also develop ascites, pleural and pericardial effusion, thromboembolism and multiple organ failure. . $^{2,4}$

The incidence of moderate-to-severe OHSS is approx. $3.1-8 \%$ of in vitro fertilization (IVF) cycles, but could be as high as $20 \%$ in high-risk women.,5,6 Monitoring serum estradiol (E2) levels was deemed effective in reducing the incidence of $\mathrm{OHSS}^{7,8}$; however, its relevance to predicting the occurrence of OHSS, especially during $\mathrm{COH}$, remains controversial. ${ }^{9,10}$ Several factors associated with OHSS have been identified, including young age, low body mass index (BMI), polycystic ovary syndrome (PCOS) or polycystic ovarian morphology, high anti-Mullerian hormone levels, a high antral follicle count (AFC), high gonadotropin ( $\mathrm{Gn}$ ) dosage, previous OHSS, high serum E2 levels, a high number of follicles or collected eggs, luteal support with human chorionic gonadotropin (hCG), and pregnancy. ${ }^{11-13}$ Since there is no specific effective treatment for OHSS, there has been considerable research aimed at identifying predisposing factors in order to predict early onset of the complication.

In this study, patients undergoing in vitro fertilization procedures by intracytoplasmic sperm injection/embryo transfer (IVF-ICSI/ET) at our reproductive center were retrospectively analyzed to explore the risk factors associated with the occurrence of severe OHSS.

\section{Materials and methods}

\section{Study population}

Between January 2013 and December 2014, a total of 350 IVF-ICSI/ET cycles were performed at our hospital. Excluding 50 cycles cancelled for cycle stimulation or with incomplete clinical data, 300 complete ovarian stimulation cycles were retrospectively analyzed. Among the 300 cycles included in this study, clinical data obtained from the 33 cycles with moderate-to-severe OHSS were grouped into the OHSS group and the other 267 cycles were grouped into the non-OHSS group. All experiments on human subjects were conducted in accordance with the Declaration of Helsinki; written informed consent was obtained from all the participants enrolled; and this study was approved by the local Ethics Committee.

\section{IVF-ICSI/ET treatment}

Gonadotropin-releasing hormone agonist (GnRH-a)/Gn/ hCG was used as treatment to induce ovulation. In this study, induction therapy included short-acting $(\mathrm{n}=183)$, long-acting $(n=65)$ and ultra-long-acting $(n=23)$ longterm treatment, micro-stimulation treatment $(\mathrm{n}=15)$, short-term treatment $(n=9)$, antagonist treatment $(n=3)$ and improved ultra-long term treatment $(n=2)$.

\section{Short- and long-acting long-term treatment}

A one-third-dose or half-dose depot of long-acting GnRH-a, or a dosage of 0.05-0.1 mg per day of short-acting GnRH-a until the day of hCG injection was administered 5-7 days after ovulation or after 15 days of Marvelon. Following 10-14 days of GnRH-a, hCG was injected when the following criteria were met: a) at least 1 follicle with a diameter of $19 \mathrm{~mm}, 2$ follicles with a diameter of $18 \mathrm{~mm}$ or 3 follicles with a diameter of $17 \mathrm{~mm}$; b) an average blood E2 level of 250-300 ng/L when each dominant follicle had a diameter of $16 \mathrm{~mm}$ or greater or at least $60 \%$ of follicles had a diameter greater than $16 \mathrm{~mm}$. The dosage of the hCG injection was 5,000-10,000 IU, and oocytes were retrieved 34-36 $\mathrm{h}$ after the injection.

\section{Short-term treatment}

Starting on the $2^{\text {nd }}$ day of the menstrual cycle, shortacting $\mathrm{GnRH}$-a was given at a dosage of $0.1 \mathrm{mg}$ per day, and on the first 2-3 days of the menstrual cycle, Gn was given until the day of hCG administration. The condition for $\mathrm{hCG}$ injection was the same as in the long-term treatment, and oocytes were retrieved $34-36 \mathrm{~h}$ after the injection.

\section{Ultra-long-term treatment}

On the $2^{\text {nd }}$ day of the menstrual cycle, the $1^{\text {st }}$ dose of longacting GnRH-a was given at a dosage of $3.75 \mathrm{mg}$, followed by a $2^{\text {nd }}$ dose after 28 days. A total of 2 or 3 subsequent doses was given at the same interval; 14-16 days after the last dose of GnRH-a, Gn was initiated at a dosage of $1.875 \mathrm{mg}$ until the day of hCG administration. The conditions for hCG injection were the same as in the long-term treatment, and oocytes were retrieved $34-36 \mathrm{~h}$ after the injection.

\section{Antagonist treatment}

On the $2^{\text {nd }}-3^{\text {rd }}$ day of the menstrual cycle, Gn treatment was initiated to induce ovulation. When a follicle with a diameter of nearly $14 \mathrm{~mm}$ or greater was observed, 
GnRH-a was given at a dosage of $0.25 \mathrm{mg}$ per day until the day of hCG administration. Again, the conditions for $\mathrm{hCG}$ injection were the same as in the long-term treatment, and oocytes were retrieved $34-36 \mathrm{~h}$ after the injection.

\section{Micro-stimulation treatment}

On the $3^{\text {rd }}$ day of the menstrual cycle, oral clomiphene citrate (50-100 mg per day) or letrozole (2.5-5 mg per day) in combination with Gn (150 IU per day) was started for a total of 5 days. On the $8^{\text {th }}$ day of the menstrual cycle, the size of the follicles was measured using vaginal ultrasonography. If the follicle diameter was less than $10 \mathrm{~mm}$, the dosage of Gn was increased by 75 IU per day. Otherwise, the dosage was maintained. Again, the condition for hCG injection was the same as in the long-term treatment, and oocytes were retrieved $34-36 \mathrm{~h}$ after the injection.

\section{Collection of the clinical data}

Prior to IVF-ICSI/ET therapy, the patients' demographics, including age, BMI, infertility duration and AFC, were collected. During the therapy, the Gn dosage, number of days of Gn treatment, the FSH, LH and E2 levels on the day of Gn treatment initiation, and the LH level, E2 level, Pg level, the total number of follicles, the number of oocytes and the number of metaphase II (MII) oocytes on the day of hCG injection were obtained. The basal antral follicular count was measured using vaginal ultrasonography between the $2^{\text {nd }}$ and $3^{\text {rd }}$ day of the menstrual cycle, and antral follicles with a diameter of 2-10 mm were counted. The number of follicles with a diameter greater than $12 \mathrm{~mm}$ was counted on the day of hCG administration using vaginal ultrasonography. MII oocytes, defined as the presence of the nuclear materials or polar body after removing the cumulus granulosa cells, were collected and counted $16-18 \mathrm{~h}$ after conventional IVF fertilization. The MII oocytes fertilized using intra-cytoplasmic sperm injection during the microinjection of spermatozoa were counted when the first polar body was observed.

\section{Diagnosis and classification of OHSS}

According to expert consensus on the diagnosis and treatment of polycystic ovarian syndrome by the Endocrinology Group of Chinese Society of Obstetrics and Gynecology, OHSS can be classified as mild, moderate, severe, and life-threatening on the basis of clinical signs and symptoms, and ultrasound and laboratory findings. In this study, only those with moderate-to-severe OHSS patients were included in the OHSS group.

\section{Statistical methods}

All data was analyzed using SPSS v. 17.0 software (SPSS Inc., Chicago, USA). Quantitative data was expressed as means \pm standard deviation (SD) and differences in parameters between the groups were compared using independent sample t-tests or $\chi^{2}$ tests as appropriate. Logistic regression was used to identify risk factors.

\section{Results}

\section{Comparison of general conditions between the OHSS and non-OHSS groups}

The differences in general conditions between the OHSS and non-OHSS patients are displayed in Table 1. The mean age of the patients in the OHSS groups was 2 years younger than in the non-OHSS group; this difference was statistically significant $(p=0.015)$. A significant statistical difference in AFC was observed between the patients in the OHSS and non-OHSS groups $(24.82 \pm 8.11$ vs $16.30 \pm 8.86, \mathrm{p}<0.001)$. The results demonstrated that patients with bilateral multiple follicular development and age less than 30 years were a high-risk population for OHSS. No statistical difference in BMI or the duration of infertility was observed between the 2 groups.

Table 1. Comparison of general conditions in the OHSS and non-OHSS groups

\begin{tabular}{|l|c|c|c|}
\multicolumn{1}{|c|}{ Variables } & $\begin{array}{c}\text { OHSS group } \\
(\mathrm{n}=33)\end{array}$ & $\begin{array}{c}\text { Non-OHSS } \\
\text { group } \\
(\mathrm{n}=267)\end{array}$ & p-value \\
\hline Age [years] & $29.79 \pm 3.45$ & $31.77 \pm 4.52$ & $0.015^{*}$ \\
\hline BMI & $21.66 \pm 2.94$ & $21.46 \pm 2.81$ & 0.706 \\
\hline Duration of infertility [years] & $3.76 \pm 2.21$ & $4.41 \pm 3.28$ & 0.267 \\
\hline AFC & $24.82 \pm 8.11$ & $16.30 \pm 8.86$ & $<0.001^{*}$ \\
\hline
\end{tabular}

*statistically significant; OHSS - ovarian hyperstimulation syndrome; $\mathrm{BMI}$ - body mass index; AFC - antral follicle count.

\section{Comparison of markers before and after treatment between the OHSS and non-OHSS groups}

The differences in marker levels between OHSS and nonOHSS groups are displayed in Table 2. The total Gn dosage used in the OHSS group was 353 IU lower than in nonOHSS group; this difference was statistically significant ( $\mathrm{p}=0.011$ ). On the day of hCG injection, serum E2 and Pg levels were significantly higher in the OHSS group than in the non-OHSS group ( $\mathrm{p}<0.001$ ) and the total number of follicles was nearly double in the OHSS group compared with the non-OHSS group ( $\mathrm{p}<0.001)$. After egg retrieval, a significantly higher numbers of oocytes and MII oocytes was observed in the OHSS group than in the non-OHSS group ( $p<0.001)$. On the day of initiation of $\mathrm{Gn}$ treatment, no significant difference was observed in the levels of FSH, LH and E2 between the groups; on the day of hCG injection, no significant difference was observed in the level of LH. 
Table 2. Comparison of pre- and post-treatment marker levels in the OHSS and non-OHSS groups

\begin{tabular}{|l|c|c|c|}
\multicolumn{1}{|c|}{ Variable } & \multicolumn{1}{|c|}{$\begin{array}{c}\text { OHSS group } \\
(\mathbf{n}=33)\end{array}$} & $\begin{array}{c}\text { Non-OHSS group } \\
(\mathbf{n}=267)\end{array}$ & p-value \\
\hline Total dosage of Gn [IU] & $1,823.48 \pm 640.58$ & $2,176.08 \pm 2,176.08$ & $0.011^{*}$ \\
\hline Number of days of Gn & $10.39 \pm 1.77$ & $10.40 \pm 2.27$ & 0.987 \\
\hline FSH level [IU/L] & On the day of Gn treatment & 0.18 \\
\hline LH level [IU/L] & $3.40 \pm 1.56$ & $4.08 \pm 2.86$ & 0.408 \\
\hline E2 level [ng/L] & $1.84 \pm 1.04$ & $2.08 \pm 1.55$ & 0.123 \\
\hline E2 level [ng/L] & $10.50 \pm 9.61$ & $14.90 \pm 15.95$ & $<0.001^{*}$ \\
\hline LH level [IU/L] & On the day of hCG injection & 0.051 \\
\hline Pg level [ng/L] & $8,476.18 \pm 5,007.85$ & $4,479.02 \pm 4,154.47$ & $0.001^{*}$ \\
\hline Total number of follicles & $1.51 \pm 0.77$ & $2.25 \pm 2.15$ & $<0.001^{*}$ \\
\hline Number of oocytes & $1.10 \pm 0.56$ & $0.82 \pm 0.46$ & $<0.001^{*}$ \\
\hline Number of MIl oocytes & $22.42 \pm 6.83$ & $12.31 \pm 7.30$ & $<0.001^{*}$ \\
\hline
\end{tabular}

*statistically significant; OHSS - ovarian hyperstimulation syndrome; E2 - estradiol; FSH - follicle stimulating hormone; Gn - gonadotropin; hCG - human chorionic gonadotropin; LH - luteinizing hormone; Pg - progesterone.

Table 3. Multivariate regression analysis results for predicting OHSS syndrome

\begin{tabular}{|l|c|c|c|c|}
\hline \multicolumn{1}{|c|}{ Variable } & $\begin{array}{c}\text { Regression } \\
\text { coefficient }\end{array}$ & $\begin{array}{c}\text { Standard } \\
\text { error }\end{array}$ & p-value & OR (95\% Cl) \\
\hline $\begin{array}{l}\text { Number of follicles on the day } \\
\text { of hCG administration }\end{array}$ & 0.11 & 0.05 & $0.011^{*}$ & $1.124(1.027-1.230)$ \\
\hline
\end{tabular}

*statistically significant; OHSS - ovarian hyperstimulation; hCG - human chorionic gonadotropin; OR - odds ratio; $95 \% \mathrm{Cl}$ - 95\% confidence interval.

Table 4. Univariate regression analysis results for risk of ovarian hyperstimulation syndrome

\begin{tabular}{|l|c|c|c|c|}
\hline \multicolumn{1}{|c|}{ Variable } & $\begin{array}{c}\text { Regression } \\
\text { coefficient }\end{array}$ & $\begin{array}{c}\text { Standard } \\
\text { error }\end{array}$ & p-value & OR (95\% Cl) \\
\hline Age [years] & -0.11 & 0.05 & $0.017^{*}$ & $0.898(0.822-0.981)$ \\
\hline BMI [kg/m²] & 0.02 & 0.06 & 0.705 & $1.025(0.903-1.163)$ \\
\hline Duration of infertility [years] & -0.08 & 0.07 & 0.267 & $0.926(0.808-1.061)$ \\
\hline Basal AFC & 0.09 & 0.02 & $<0.001^{*}$ & $1.090(1.051-1.131)$ \\
\hline Total Gn dosage [IU] & 0.00 & 0.00 & $0.011^{*}$ & $0.999(0.999-1.000)$ \\
\hline & On the day of Gn treatment & \\
\hline Number of treatment days & -0.00 & 0.08 & 0.987 & $0.999(0.848-1.176)$ \\
\hline FSH level [IU/L] & -0.14 & 0.10 & 0.178 & $0.872(0.867-1.577)$ \\
\hline LH level [IU/L] & -0.12 & 0.15 & 0.408 & $0.886(0.636-1.669)$ \\
\hline E2 level [ng/L] & -0.03 & 0.02 & 0.122 & $0.969(0.909-1.010)$ \\
\hline & On the day of hCG injection & & \\
\hline E2 level [ng/L] & 0.00 & 0.00 & $<0.001^{*}$ & $1.000(1.000-1.000)$ \\
\hline LH level [IU/L] & -0.39 & 0.19 & 0.051 & $0.685(0.551-1.467)$ \\
\hline Pg level [ng/L] & 1.02 & 0.34 & $0.002^{*}$ & $2.773(0.510-3.370)$ \\
\hline Total number of follicles & 0.17 & 0.03 & $<0.001^{*}$ & $1.185(1.027-1.230)$ \\
\hline Number of oocytes & 0.23 & 0.04 & $<0.001^{*}$ & $1.254(0.894-1.472)$ \\
\hline Number of MII oocytes & 0.21 & 0.04 & $<0.001^{*}$ & $1.238(0.747-1.217)$ \\
\hline
\end{tabular}

*statistically significant; OR - odds ratio; 95\% Cl - 95\% confidence interval; OHSS - ovarian hyperstimulation syndrome; BMI - body mass index; AFC - antral follicle count; E2 - estradiol; FSH - follicle stimulating hormone; Gn - gonadotropin; hCG - human chorionic gonadotropin; $\mathrm{LH}$ - luteinizing hormone; $\mathrm{Pg}$ - progesterone.

\section{Logistic regression analysis of risk factors for OHSS}

The univariate logistic regression analysis demonstrated that age and the total dose of gonadotropin were protective factors, whereas baseline AFC, E2 and Pg levels, the total number of follicles, and the numbers of oocytes and MII oocytes on the day of hCG injection were risk factors for OHSS. Other parameters were not associated with the occurrence of OHSS. In a multivariate model using logistic stepwise regression analysis, the total number of follicles was selected as an independent risk factor for OHSS (Table 3). When the total follicle count was more than 17 on the day of hCG injection, the sensitivity and specificity of predicting the occurrence of OHSS were $84.85 \%$ and $77.15 \%$, respectively, according to the model. Details of these results are presented in Table 4.

\section{ROC curve of predicting} the occurrence of OHSS

\section{by the total number of follicles on the day of hCG injection}

The area under the ROC curve was 0.847 (95\% CI $=0.775-0.919)$ for predicting the occurrence of severe OHSS with measuring the total number of follicles on the day of hCG administration (Fig. 1). An optimal cut-off value of 17 was determined with the ROC curve analysis.

\section{Discussion}

Ovarian hyperstimulation syndrome is a complication caused by the stimulation of ovarian cycles arising from medical treatment with gonadotropin for inducing follicular maturation. With the increasing use of assisted reproductive techniques and ovulationinduction drugs, there is a growing trend for the occurrence of OHSS. ${ }^{14}$ During IVF treatment, the incidence of moderate-to-severe OHSS was approx. $3.1-8 \%$, but could reach $20 \%$ 


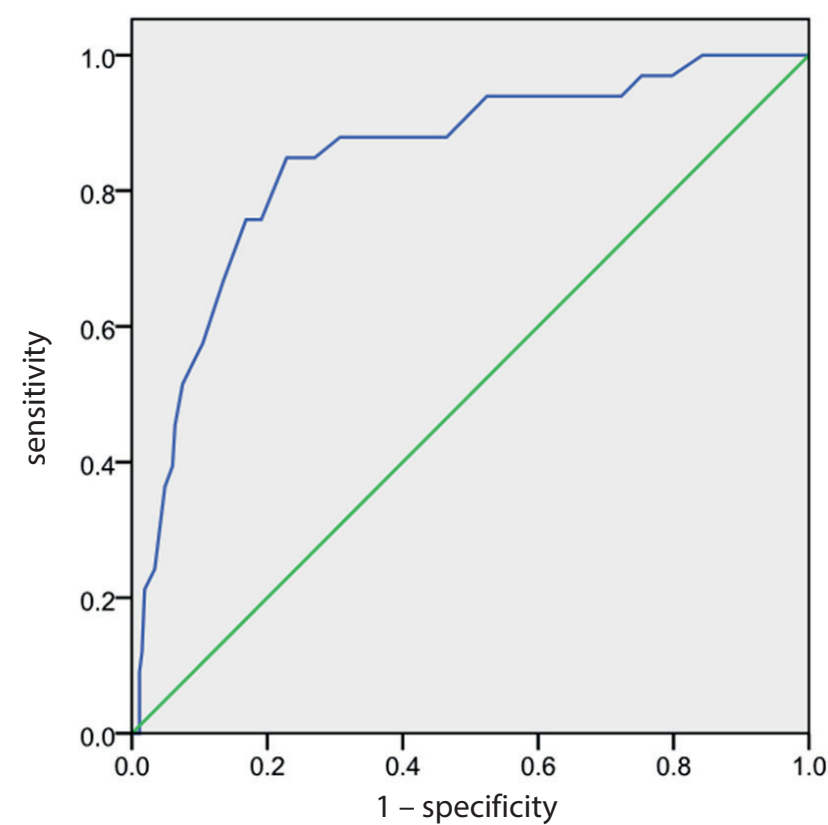

Fig. 1. Receiver operating characteristic (ROC) curve for predicting the occurrence of OHSS by the total number of follicles on the day of hCG injection

in high-risk women. ${ }^{2,5,6}$ Severe OHSS is life-threatening and could lead to hospitalization in $0.1-2 \%$ of IVF cases. ${ }^{15,16}$ Although the pathogenesis of OHSS is still unclear, VEGF, renin-angiotensin system and other cytokines may be involved. It is now recognized that hCG exposure is a risk factor for OHSS. During controlled ovarian stimulation (COS), injection of hCG induces follicular maturation and stimulates LH to the peak level. However, a high concentration of exogenous hCG exposure is considered to be a key factor in the onset of early OHSS, whereas late OHSS is mainly associated with endogenous hCG produced by corpus luteum. ${ }^{17,18}$ Currently, there is lack of effective clinical treatment for OHSS, but mainly preventive measures.

\section{Relationship between ovarian reserve and moderate and severe OHSS}

Age and AFC are reliable indicators of ovarian reserve function. Our study demonstrated that patients at a younger age (less than 30 years) and with higher AFC $(>24)$, which indicated that they had better ovarian reserve function, had a higher chance of experiencing moderate or severe OHSS in COS. These findings were consistent with a report presented at the 2013 European Society of Human Reproduction and Embryology (ESHRE) annual conference that patients with PCOS are at high risk for OHSS. ${ }^{19}$ These patients had multiple antral follicles, increased numbers of follicles and high gonadotropin receptor densities, and therefore became more responsive to gonadotropin and subsequently more prone to the occurrence of OHSS.

\section{Relationship between the use of $\mathrm{Gn}$ for COS in IVF cycles and the occurrence of OHSS}

During COS in women undergoing the IVF-ICSI/ET cycle, in order to select more dominant follicles, the use of exogenous $\mathrm{Gn}$, which increases the number of follicles, overcomes the inherent selectivity of a single dominant follicle so that multiple follicles can grow and become mature or near mature at the same time. In this study, the average total dosage of $\mathrm{Gn}$ in the OHSS group $(1,823.48 \pm 640.58 \mathrm{IU})$ was $353 \mathrm{IU}$ lower than that in the non-OHSS group $(2,176.08 \pm 2,176.08 \mathrm{IU})$, but the duration of the use of $\mathrm{Gn}$ did not reveal any significant difference. This demonstrated that patients in the OHSS group have better ovarian reserves: Although they required fewer dosage of $\mathrm{Gn}$, more follicles were obtained within the same duration of Gn usage as in the non-OHSS group. This suggested that pre-COS assessment of the ovarian reserve function is important and when appropriate follicles were obtained from young PCOS patients, the initial dosage and the duration of Gn usage should be reduced in order to prevent the occurrence of OHSS.

\section{Relationship between the total number of follicles, E2 and Pg levels, the number of oocytes and MII oocytes, and the occurrence of OHSS}

During an IVF cycle, GnRH-a is used for downregulation to increase the number of follicles obtained. Following the use of $\mathrm{Gn}$ for a period of time to obtain the dominant follicle, exogenous hCG is injected to promote final follicular maturation. Therefore, the total number of follicles and E2 levels on the day of hCG injection is critical. In our study, it was found that the total number of follicles with diameters greater than $12 \mathrm{~mm}$ was $22.42 \pm 6.83$ in the OHSS group, which was significantly more than the number in the non-OHSS group (12.31 \pm 7.30$)$. At our center, hCG injection was applied by the number of dominant follicles obtained. As a result, there were not many dominant follicles although the total number of follicles obtained was higher in the OHSS group and the number of days of Gn use were basically the same as in the nonOHSS group. This increased the probability of OHSS occurrence. On the other hand, in this study, the E2 and Pg levels and the number of oocytes and MII oocytes on the day of hCG injection were significantly different between the OHSS and non-OHSS groups. According to the multivariate logistic regression analysis, the total number of follicles on the day of hCG administration was the only predictive factor for the occurrence of OHSS. An optimal cut-off value of 17 was determined by the ROC curve analysis. In other words, when the total number of follicles was greater than 17 , the sensitivity and specificity of the model in predicting the occurrence of moderate 
and severe OHSS were $84.85 \%$ and $77.15 \%$, respectively. Kwan et al. ${ }^{20}$ and Aramwit et al. ${ }^{21}$ reported that the E2 level and the number of oocytes retrieved can effectively predict the occurrence of OHSS. In our study, although both E2 levels and the number of oocytes were statistically different between the OHSS and non-OHSS groups, the multivariate logistic regression analysis did not reveal them as independent predictive factors. This may possibly be because the highest measurable value for the E2 marker was set at 4,300 $\mathrm{ng} / \mathrm{L}$, requiring dilution for measurement, which may have introduced a measurement error. However, in clinical settings, it is very frequent that the levels of E2 and the number of follicles do not match. As for the number of oocytes, since it depends on the surgical skills of doctors and the condition of the patients, it may not fully represent the total number of follicles, especially in patients who have difficulty in egg retrieval.

In summary, the multivariate logistic regression analysis revealed that the total number of follicles on the day of hCG administration is an independent predictive factor for OHSS. These findings could guide clinicians regarding the timing and dosage for the use of Gn during COS. When the total number of follicles exceeds 17, appropriate measures including $\mathrm{Gn}$ dose reduction, hCG administration or GnRH-a injection should be taken, and patients should be recommended for embryo cryopreservation to prevent the occurrence of OHSS.

\section{References}

1. Nastri CO, Ferriani RA, Rocha IA, Martins WP. Ovarian hyperstimulation syndrome: Pathophysiology and prevention. J Assist Reprod Genet. 2010;27(2-3):121-128.

2. Nastri CO, Teixeira DM, Moroni RM, Leitão VM, Martins WP. Ovarian hyperstimulation syndrome: Pathophysiology, staging, prediction and prevention. Ultrasound Obstet Gynecol. 2015;45(4):377-393.

3. Kaiser UB. The pathogenesis of the ovarian hyperstimulation syndrome. N Engl J Med. 2003;349(8):729-732.

4. Busso C, Fernández-Sánchez M, García-Velasco JA, et al. The non-ergot derived dopamine agonist quinagolide in prevention of early ovarian hyperstimulation syndrome in IVF patients: A randomized, doubleblind, placebo-controlled trial. Hum Reprod. 2010;25(4):995-1004.

5. Delvigne A, Rozenberg S. Epidemiology and prevention of ovarian hyperstimulation syndrome (OHSS): A review. Hum Reprod Update. 2002;8(6):559-577.
6. Smith V, Osianlis T, Vollenhoven B. Prevention of ovarian hyperstimulation syndrome: A review. Obstet Gynecol Int. 2015;2015:514159.

7. Varma TR, Patel RH. Ovarian hyperstimulation syndrome: A case history and review. Acta Obstet Gynecol Scand. 1988;67(7):579-584.

8. Golan A, Ron-el R, Herman A, Soffer Y, Weinraub Z, Caspi E. Ovarian hyperstimulation syndrome: An update review. Obstet Gynecol Surv. 1989:44(6):430-440.

9. Aboulghar M. Prediction of ovarian hyperstimulation syndrome (OHSS): Estradiol level has an important role in the prediction of OHSS. Hum Reprod. 2003;18(6):1140-1141.

10. Orvieto R. Prediction of ovarian hyperstimulation syndrome: Challenging the estradiol mythos. Hum Reprod. 2003;18(4):665-667.

11. König E, Bussen S, Sütterlin M, Steck T. Prophylactic intravenous hydroxyethyle starch solution prevents moderate-severe ovarian hyperstimulation in in-vitro fertilization patients: A prospective, randomized, double-blind and placebo-controlled study. Hum Reprod. 1998;13(9):2421-2424.

12. Nargund G, Hutchison L, Scaramuzzi R, Campbell S. Low-dose HCG is useful in preventing OHSS in high-risk women without adversely affecting the outcome of IVF cycles. Reprod Biomed Online. 2007;14(6): 682-685.

13. O'Donovan O, Chami AA, Davies M. Ovarian hyperstimulation syndrome. Obstet Gynaecol Reprod Med. 2015;25(2):43-48.

14. de Mouzon J, Goossens V, Bhattacharya S, et al; European IVF-monitoring (EIM) Consortium, for the European Society of Human Reproduction and Embryology (ESHRE). European IVF-monitoring (EIM) Consortium, for the European Society of Human Reproduction and Embryology (ESHRE): Assisted reproductive technology in Europe, 2006: Results generated from European registers by ESHRE. Hum Reprod. 2010;25(8):1851-1862.

15. Aboulghar MA, Mansour RT. Ovarian hyperstimulation syndrome: Classifications and critical analysis of preventive measures. Hum Reprod Update. 2003;9(3):275-289.

16. Mocanu E, Redmond ML, Hennelly B, Collins C, Harrison R. Odds of ovarian hyperstimulation syndrome (OHSS): Time for reassessment. Hum Fertil (Camb). 2007;10(3):175-181.

17. Mathur RS, Akande AV, Keay SD, Hunt LP, Jenkins JM. Distinction between early and late ovarian hyperstimulation syndrome. Fertil Steril. 2000;73(5):901-907.

18. Humaidan P, Quartarolo J, Papanikolaou EG. Preventing ovarian hyperstimulation syndrome: Guidance for the clinician. Fertil Steril. 2010;94(2):389-400.

19. Abstracts of the $29^{\text {th }}$ Annual Meeting of the European Society of Human Reproduction and Embryology. London, UK. July 7-10, 2013. Hum Reprod. 2013;28(Suppl 1):370.

20. Kwan I, Bhattacharya S, Kang A, Woolner A. Monitoring of stimulated cycles in assisted reproduction (IVF and ICSI). Cochrane Database Syst Rev. 2014;8:CD005289.

21. Aramwit P, Pruksananonda K, Kasettratat N, Jammeechai K. Risk factors for ovarian hyperstimulation syndrome in Thai patients using gonadotropins for in vitro fertilization. Am J Health Syst Pharm. 2008; 65(12):1148-1153. 